\section{Multiple neoplasms within a single adrenal gland of a dog}

\section{Tom Hernon, Katie McCallum, Laura Owen}

Queen's Veterinary School Hospital, University of Cambridge, Cambridge, United Kingdom

\section{OBJECTIVES}

To present a canine patient with multiple neoplasms identified within a single adrenal gland and to discuss the diagnostics and management of the condition.

\section{METHODS}

A rare canine patient with multiple neoplasms present within a single adrenal gland is described.

\section{RESULTS}

An 11-year-old neutered female Labrador retriever was presented with a history of tachypnoea, polyuria and polydipsia. Diagnostics included haematology, biochemistry, a low-dose dexamethasone suppression test, plasma ACTH, urine metanephrine and normetanephrine, serial Doppler blood pressure measurements and computed tomography (CT).

CT identified a large bi-lobed abdominal mass in the location of the left adrenal gland. The results of endocrine testing were consistent with adrenal-dependant hyperadrenocorticism, although the presence of intermittent hypertension and borderline high metanephrines were suggestive of a concurrent phaeochromocytoma.

As a phaeochromocytoma could not be ruled out, preparatory steps were taken preoperatively including ensuring appropriate drugs were available, pre-emptive blood typing and arterial blood pressure monitoring. A unilateral adrenalectomy was performed. Histopathological examination identified a benign myelolipoma and two distinct malignancies within the adrenal gland; a phaeochromocytoma and an adrenocortical adenocarcinoma. Postoperative recovery was uneventful and resolution of all clinical signs was observed within 4 weeks. No signs of recurrence or metastatic disease were noted 5 months postoperatively. The patient was euthanised 12 months postoperatively due to metastatic disease.

\section{STATEMENT (CONCLUSIONS)}

Multiple unilateral adrenal neoplasms are rare. This case demonstrates the importance of considering the presence of multiple functional tumours in a single patient and performing detailed endocrine testing to optimise pre/perioperative stabilisation and outcome. 